\title{
A non-ordinary state-based peridynamics formulation for thermoplastic fracture
}

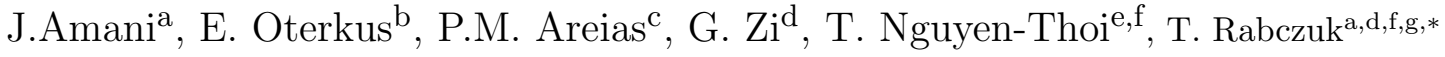 \\ ${ }^{a}$ Institute of Structural Mechanics, Bauhaus-Universität Weimar, Marien-Str. 15, 99423 Weimar, Germany \\ ${ }^{b}$ Department of Naval Architecture, Ocean and Marine Engineering, University of Strathclyde, Glasgow, UK \\ ${ }^{c}$ Department of Physics, Colgio Lus Antnio Verney, University of vora, Rua Romo Ramalho, 59, vora, Portugal \\ ${ }^{d}$ Korea University, School of Civil, Environmental and Architectural Engineering, Seoul, South Korea \\ ${ }^{e}$ Division of Computational Mathematics and Engineering (CME), Institute for Computational Science (INCOS), Ton Duc \\ Thang University, VietNam \\ ${ }^{f}$ Faculty of Civil Engineering, Ton Duc Thang University, Ho Chi Minh City, VietNam \\ ${ }^{g}$ Division of Computational Mechanics (DCM), Ton Duc Thang University, Ho Chi Minh City, Vietnam
}

\begin{abstract}
In this study, a three-dimensional (3D) non-ordinary state-based peridynamics (NOSB-PD) formulation for thermomechanical brittle and ductile fracture is presented. The Johnson-Cook (JC) constitutive and damage model is used to taken into account plastic hardening, thermal softening and fracture. The formulation is validated by considering two benchmark examples: 1) The Taylor-bar impact and 2) the Kalthoff-Winkler tests. The results show good agreements between the numerical simulations and the experimental results.
\end{abstract}

Keywords: Non-local model, Peridynamics, Thermoplasticity, Johnson-Cook model, Fracture.

\section{Introduction}

For numerous years, materials and structures with thermomechanical characteristic have attracted significant interest. The presence of defects, such as notches and voids, reduce its functionality because of initiation and propagation of cracks. Despite the existence of many approaches for numerical simulation of crack initiation and propagation in local theory of continuum mechanics (e.g. extended finite element [1,2], meshfree $[3,4,5,6,7,8,9]$, phantom-node [10, 11, 12], XIGA [13, 14] and remeshing techniques [15, 16]), it is still a major challenge within these frameworks. From the mathematical point of view, they are based on partial differential equations in which spatial derivatives fail whenever a discontinuity appears in a body

\footnotetext{
* Corresponding author

Email addresses: jafar.amani.dashlejeh@uni-weimar.de (J.Amani), erkan.oterkus@strath.ac.uk (E. Oterkus), pmaa@uevora.pt (P.M. Areias), g-zi@korea.ac.kr (G. Zi), nguyenthoitrung@tdt.edu.vn (T. Nguyen-Thoi), timon.rabczuk@uni-weimar.de (T. Rabczuk)
} 
[17]. The domain of the applicability of classical field theories is intimately connected to the length and time scales. If $L$ denotes the external characteristic length (e.g. crack length, wavelength) and $l$ the internal characteristic length (e.g. granular distance, lattice parameter), then in the region $L / l>>1$, classical field theories predict sufficiently accurate results. On the other hand, when $L / l \sim 1$, local theories fail. In dynamics, there will be a similar scale $T / t$ where $T$ is the external characteristic time (e.g. the time scale of the applied load) and $t$ is the internal characteristic time (e.g. the time scale of signal transmission from one molecule to the next). Again, classical theories fail when $T / t \sim 1$. Hence, the physical phenomenon in space and time scales requires nonlocality scaled by $L / l$ and $T / t$ [18]. Nonlocal continuum theory can be used to overcome the above mathematical challenge of the local theories. The concept of nonlocality is inherent in solid state physics where the nonlocal attractions of atoms are prevalent. Here, the material is considered to consist of discrete atoms connected by distant forces from other neighboring atoms [18].

A nonlocal formulation based on integro-differential equations called peridynamics (PD) was introduced by Silling [19]. The PD theory reformulates the equation of motion such that no spatial derivatives are required. In the PD theory, a continuous body consists of material points interacting in a nonlocal manner. Hence, PD can be considered as a nonlocal meshless method with advantages in problems involving large deformation where mesh-based methods fail. The PD theory permits modeling bodies with discontinuities and its propagation. Fracture is the natural outcome of the PD simulation and damage is a part of the material response. The original version of PD [19] later was named as the bond-based peridynamics (BB-PD) theory. The state-based PD (SB-PD) theory [20] is a generalization of the BB-PD theory (for limitations of the BB-PD see [20]). The SB-PD has two types: ordinary state-based (OSB) and non-ordinary statebased (NOSB) (for details about the differences ref to the definition 8.4 in [20]). To establish a SB-PD, the material-dependent part has been rewritten, introducing a mathematical object called a "force-vector state" that is in some ways similar to the traditional stress tensor of classical continuum mechanics. Therefore, the traditional constitutive models can be incorporated into the SB-PD model. For instance, Mitchell [21] implemented an elastic-perfect plasticity constitutive model into the OSB-PD theory. Similar contribution the OSB-PD includes in the work by Warren et al. [22] for incorporating the elasto-plastic linear hardening constitutive model. In the NOSB-PD theory, Foster et al. [23] extended viscoplastic material model and Tupek et al. [24] incorporated the modified Johnson-Cook constitutive model that does not contain the temperature effect.

The PD theory was extended successfully to heat diffusion problems. Kilic and Madenci [25] used the BB-PD theory for prediction of thermally driven crack propagation patterns in quenched glass plates containing single or multiple pre-existing cracks due to thermal loading. Bobaru and Duangpanya [26] formulated a one-dimensional BB-PD theory for the heat conduction equation and also extended it to solve two-dimensional problems with discontinuities [27]. Kilic and Madenci [28] proposed a BB-PD formulation for uncoupled thermomechanical problems. They included the thermal term in the response function of 
the PD interactions. They introduced a multi-dimensional PD heat conduction equation and considered domains with discontinuities such as insulated cracks. Gerstle et al. [29] developed a BB-PD model for electro-migration that accounts for one-dimensional heat conduction problems. Oterkus et al. [30] derived a SB-PD model of the heat conduction equation based on the Lagrangian formalism. Also, Oterkus et al. [31] extended it for analysis of 2D problems. Agwai [32] formulated an OSB-PD formulation for fully coupled thermoelasticity using the conservation of thermal and mechanical energy along with the freeenergy function. She also derived a dimensionless BB-PD form of the coupled equations using the OSB-PD formulation and applied it to solve one dimensional examples. Oterkus et al. [33] extended it to solve 2D and 3D problems.

To our best knowledge, no attempt has been made to develop the NOSB-PD theory for ductile fracture analysis of thermoplasticity problems. Hence, the main purpose and motivation of this paper is to extend a 3D thermomechanical model to a NOSB-PD framework to use its ability to deal with ductile fracture analysis. One of the main feature of ductile fracture in thermomechanical process is its dissipative character. The large amount of heat generated by plastic work around the crack tip area causes thermal softening, shear banding and thermal damage [34]. Among the most popular constitutive models for ductile fracture accounting for plastic hardening, thermal softening, strain rate effects and ductile damage is the JohnsonCook model. For adiabatic heating, the thermomechanical coupling can be modeled only through the inelastic heat fraction which indicates the plastic work fraction converted into heat. We will restrict our attention to thermoplasticity problems subjected to adiabatic heating and it is not our purpose to solve the fully coupled thermomechanical equations. We adopt the JC model for constitutive modeling and for damage assessment. We present the detailed implementation of the NOSB-PD for thermoplasticity. The Taylor-bar impact test is exploited to validate the proposed method. Another validation experiment is the Kalthoff-Winkler experiment.

The remaining of this paper is organized as follows: Section 2 provides the JC constitutive and damage model. In section 3, the concept of the PD theory and also the NOSB-PD formulation of the thermomechanical problems are presented. The numerical verification and results are presented and compared against experimental data in section 4. Finally, some concluding remarks are summarized.

\section{Johnson-Cook model}

\subsection{Johnson-Cook constitutive model}

In dynamic applications such as high velocity impact problems, the effects of strain hardening, thermal softening and strain rate are important. The Johnson-Cook constitutive model $[35,36]$ accounts for those 
effects. The von-Mises flow stress from the JC constitutive model is given by

$$
S=\left[A+B \epsilon_{p}^{n}\right]\left[1+C \ln \dot{\epsilon}_{p}^{*}\right]\left[1-T^{* m}\right], \quad \dot{\epsilon}_{p}^{*}=\frac{\dot{\epsilon}_{p}}{\dot{\epsilon}_{p_{0}}},
$$

where $\epsilon_{p}$ is the equivalent plastic strain, $A$ is the yield stress, $B$ and $n$ are strain hardening constants, $C$ is the strain rate constant, $m$ is the temperature softening exponent parameter, $\dot{\epsilon}_{p}^{*}$ is the dimensionless plastic strain rate, $\dot{\epsilon}_{p}$ and $\dot{\epsilon}_{p_{0}}=1.0 \mathrm{~s}^{-1}$ are the plastic strain rate and its reference value, respectively and $T^{*}$ is the homologous temperature defined as

$$
T^{*}=\frac{T-T_{0}}{T_{m}-T_{0}}
$$

in which $T, T_{0}$ and $T_{m}$ are the current, reference (room) and melting temperatures, respectively.

Camacho and Ortiz [37] modified the von-Mises flow stress of the JC constitutive model to avoid unwanted effects for $\dot{\epsilon}_{p}^{*}<1$. Therefore,

$$
S=\left[A+B \epsilon_{p}^{n}\right]\left[1+\dot{\epsilon}_{p}^{*}\right]^{C}\left[1-T^{* m}\right]
$$

\subsection{Johnson-Cook damage model}

The Johnson-Cook damage model relates the accumulative damage $D$ to an equivalent plastic strain increment $\Delta \epsilon_{p}$ as [36]

$$
D=\sum_{k=1}^{n t} \frac{\Delta \epsilon_{p}}{\epsilon^{f}},
$$

where $n t$ is the number of time steps in the time discretization procedure and $\epsilon^{f}$ is the fracture strain defined as a function of the homologous temperature, strain rate and pressure as [36, 38]:

$$
\epsilon^{f}=\left[D_{1}+D_{2} \exp \left(D_{3} \sigma^{*}\right)\right]\left[1+D_{4} \ln \dot{\epsilon}_{p}^{*}\right]\left[1+D_{5} T^{*}\right], \quad \sigma^{*}=\frac{\sigma_{\mathrm{m}}}{S} \leq 1.5,
$$

where $D_{1}, \ldots, D_{5}$ are material constants, $\sigma_{\mathrm{m}}=\operatorname{trace}(\boldsymbol{\sigma}) / 3$ is the hydrostatic pressure obtained from the Cauchy stress tensor $\sigma$ and $\sigma^{*}$ is the stress triaxiality ratio. When $\sigma^{*}>1.5$, a linear relationship for the fracture strain $\epsilon^{f}$ was proposed in [36]. The $\sigma_{\text {spall }}^{*}=\sigma_{\text {spall }} / S$ is the dimensionless spall fracture computed from the spall fracture and the current value of the von-Mises stress. The first bracket of Eq. (5) reveals that the fracture strain decreases as the hydrostatic pressure increases. The second and third brackets account for strain rate and temperature effects, respectively. $D=0$ indicates the undamaged material point while $D=1$ indicates the material point is fully damaged. 
In order to avoid unwanted effects for $\dot{\epsilon}_{p}^{*}<1$ the fracture strain is modified as [39]

$$
\epsilon^{f}=\left[D_{1}+D_{2} \exp \left(D_{3} \sigma^{*}\right)\right]\left[1+\dot{\epsilon}_{p}^{*}\right]^{D_{4}}\left[1+D_{5} T^{*}\right], \quad \sigma^{*}=\frac{\sigma_{\mathrm{m}}}{S} \leq 1.5 .
$$

Hence, the JC constitutive model by considering damage can be written as [39]

$$
S=(1-D)\left[A+B \epsilon_{r p}^{n}\right]\left[1+\dot{\epsilon}_{r p}^{*}\right]^{C}\left[1-T^{* m}\right], \quad \dot{\epsilon}_{r p}^{*}=\frac{\dot{\epsilon}_{r p}}{\dot{\epsilon}_{p_{0}}},
$$

where $\epsilon_{r p}$ is the damage accumulated plastic strain, defined in the rate form by $\dot{\epsilon}_{r p}=(1-D) \dot{\epsilon}_{p}$ and $\dot{\epsilon}_{r p}^{*}$ is the dimensionless plastic strain rate for the damaged material point.

\section{Peridynamics theory of thermomechanics}

\subsection{Governing thermomechanical equations}

The strong form of the linear momentum balance is

$$
\rho_{0} \frac{\partial^{2} \mathbf{u}}{\partial t^{2}}=\nabla \cdot \mathbf{P}+\mathbf{b}
$$

and the strong form of the energy balance is

$$
\rho_{0} C_{p} \frac{\partial T}{\partial t}=\beta \boldsymbol{\tau}: \mathbf{d}^{p}+\nabla\left(J \mathbf{F}^{-1} \cdot \mathbf{K}_{T} \cdot \mathbf{F}^{-T} \cdot \nabla T\right)
$$

where $\rho_{0}$ is the initial mass density, $\mathbf{u}$ is the displacement vector and $t$ is the time; $C_{p}$ is the specific heat capacity, $T$ is the temperature and $\beta$ is the Taylor-Quinney empirical constant [40] represent fraction of the total plastic work that is converted into heat. It is indicated that this parameter vary with plastic strain [41]. It is commonly assumed that $90-100 \%$ of the plastic work is converted into heat; $\boldsymbol{\tau}$ is the Kirchhoff stress tensor and $\mathbf{d}^{p}$ is the plastic part of the deformation rate tensor $\mathbf{d} ; \mathbf{F}$ is the deformation gradient tensor; $\mathbf{K}_{T}=\kappa \mathbf{1}$ is the heat conductivity tensor for isotropic materials where $\kappa$ is the heat conductivity coefficient and $\mathbf{1}$ is the second order unit tensor, $\mathbf{P}$ is the first Piola-Kirchhoff stress tensor and $J=\operatorname{det}(\mathbf{F})>0$ is the determinant of the deformation gradient tensor.

These two equations are coupled intrinsically through the constitutive equations. In general, the numerical simulation of such coupled systems is complex due to different time-scales associated with thermal and mechanical fields [42]. For solving these equations two numerical integration schemes namely monolithic $[43,44,45]$ and operator splitting $[46,47]$ techniques can be employed. In the operator splitting technique Eq. (9) can be split into two sub-system [34]: (a) heat generation due to an adiabatic mechanical system 
(a)

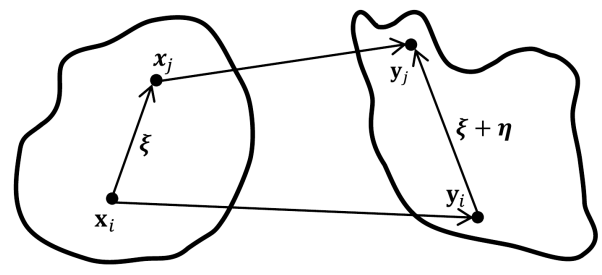

(b)

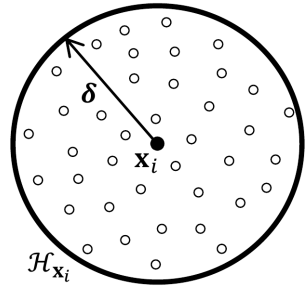

Figure 1. (a) Reference and deformed vector states, (b) Horizon $\mathcal{H}_{\mathbf{x}_{i}}$ with radius $\delta$ centered at $\mathbf{x}_{i}$.

(plastic deformation) and (b) heat conduction based system and can be shown as:

$$
\begin{cases}\text { Adiabatic heating : } & \rho_{0} C_{p} \frac{\partial T}{\partial t}=\beta \boldsymbol{\tau}: \mathbf{d}^{p}, \\ \text { Heat conduction : } & \rho_{0} C_{p} \frac{\partial T}{\partial t}=\nabla\left(J \mathbf{F}^{-1} \cdot \mathbf{K}_{T} \cdot \mathbf{F}^{-\mathrm{T}} \cdot \nabla T\right), \quad(\mathrm{b}),\end{cases}
$$

For solving Eq. (10) the energy balance equation is integrated in two steps [48]:

1. updating the temperature due to adiabatic heating in the JC constitutive model,

2. updating the temperature change due to heat conduction in the main time integration loop.

Eq. (10)-(b) needs to be reformulated into integro-differential form for updating the temperature change due to heat conduction in a PD framework. Since high velocity impact problems occur over a short time (a few hundred $\mu s$ ), we only considered the heat change due to the effect of adiabatic heating and the effect of heat conduction may be negligible [3]. It is commonly assumed that plastic energy is transferred into heat and the heat change is calculated as:

$$
\Delta T=\frac{\beta}{\rho_{0} C_{p}} \int_{0}^{\epsilon_{p}} S d \epsilon_{p} .
$$

Here, the coupled thermo-viscoplastic problem is reduced to a mechanical problem [3]. So, the momentum equation, Eq. (8), suffices for constructing the NOSB formulation, and the energy equation, Eq. (10)-(a), is only used in the constitutive update.

\subsection{Peridynamic states}

Let the spatial position vectors of material point $i$ and $j$ in the reference (initial) configuration $\Omega_{0}$ be $\mathbf{x}_{i}$ and $\mathbf{x}_{j}$, respectively (see Fig. 3.2-(a)), $\mathbf{u}\left(\mathbf{x}_{i}, t\right)$ and $\mathbf{u}\left(\mathbf{x}_{j}, t\right)$ denote the displacement vectors of the material points $i$ and $j$ at time $t$ in the deformed configuration $\Omega$, respectively, $\mathbf{y}\left(\mathbf{x}_{i}, t\right)$ and $\mathbf{y}\left(\mathbf{x}_{j}, t\right)$ are position vectors of the material points $i$ and $j$ at time $t$ in the deformed configuration $\Omega$, respectively. The state of the material point $\mathbf{x}_{i}$ is determined by its interaction with the material points that are located within a finite distance $\mathcal{H}_{\mathbf{x}_{i}}$ called the horizon with radius $\delta$ centered at $\mathbf{x}_{i}$. The material point $i$ interacts nonlocally with all material points $j$ that lie within $\mathcal{H}_{\mathbf{x}_{i}}$ (see Fig. $3.2-(\mathrm{b})$ ). 
Some specific states that are useful in the PD concept are :

$$
\begin{aligned}
& \text { reference position vector state : } \underline{\mathbf{X}}\langle\boldsymbol{\xi}\rangle=\boldsymbol{\xi}=\mathbf{x}_{j}-\mathbf{x}_{i} \text {, } \\
& \text { displacement vector state : } \underline{\mathbf{U}}[\mathbf{x}, t]\langle\boldsymbol{\xi}\rangle=\boldsymbol{\eta}=\mathbf{u}\left(\mathbf{x}_{j}, t\right)-\mathbf{u}\left(\mathbf{x}_{i}, t\right) \text {, } \\
& \text { deformation vector state : } \underline{\mathbf{Y}}[\mathbf{x}, t]\langle\boldsymbol{\xi}\rangle=\boldsymbol{\xi}+\boldsymbol{\eta}=\mathbf{y}\left(\mathbf{x}_{j}, t\right)-\mathbf{y}\left(\mathbf{x}_{i}, t\right) \text {, }
\end{aligned}
$$

where $\underline{\mathbf{X}}\langle\boldsymbol{\xi}\rangle$ shows the state $\underline{\mathbf{X}}$ acting on $\boldsymbol{\xi}$. A vector state is the generalization of a second-rank tensor, i.e. the state $\underline{\mathbf{X}}$ maps the reference position vector state $\boldsymbol{\xi}$ to a vector in $\mathbb{R}^{3}[20], \underline{\mathbf{Y}}[\mathbf{x}, t]$ indicates the state $\underline{\mathbf{Y}}$ depends on the spatial position vector $\mathbf{x}$ and time $t$.

\subsection{A non-ordinary state-based peridynamics formulation of thermoplasticity}

The equation of motion in the state-based PD can be formulated as [20]

$\rho_{0}\left(\mathbf{x}_{i}\right) \ddot{\mathbf{u}}\left(\mathbf{x}_{i}, t\right)=\int_{\mathcal{H}_{\mathbf{x}_{i}}}\left\{\underline{\mathbf{T}}[\mathbf{x}, t]\left\langle\mathbf{x}_{j}-\mathbf{x}_{i}\right\rangle-\underline{\mathbf{T}}[\mathbf{x}, t]\left\langle\mathbf{x}_{i}-\mathbf{x}_{j}\right\rangle\right\} d V_{\mathbf{x}_{j}}+\mathbf{b}\left(\mathbf{x}_{i}, t\right), j=1,2, \ldots, m, \forall i=1,2, \ldots, N$,

where $N$ represents the total number of nodes used for discretization of the body, $m$ is the number of material points in the horizon of the material point $\mathbf{x}_{i}$ and $\mathbf{b}$ is an external body force density vector, $\underline{\mathbf{T}}[\mathbf{x}, t]\left\langle\mathbf{x}_{j}-\mathbf{x}_{i}\right\rangle$ and $\underline{\mathbf{T}}[\mathbf{x}, t]\left\langle\mathbf{x}_{i}-\mathbf{x}_{j}\right\rangle$ are the force-vector states. Conditions on $\underline{\mathbf{T}}$ for which Eq. (15) satisfies the balance of linear and angular momentum were given in [20].

In order to discretize Eq. (15), the integral is approximated with a finite sum as

$\rho_{0}\left(\mathbf{x}_{i}\right) \ddot{\mathbf{u}}\left(\mathbf{x}_{i}, t\right) \approx \sum_{j=1}^{m}\left\{\underline{\mathbf{T}}[\mathbf{x}, t]\left\langle\mathbf{x}_{j}-\mathbf{x}_{i}\right\rangle-\underline{\mathbf{T}}[\mathbf{x}, t]\left\langle\mathbf{x}_{i}-\mathbf{x}_{j}\right\rangle\right\} V_{\mathbf{x}_{c j}}+\mathbf{b}\left(\mathbf{x}_{i}, t\right), j=1,2, \ldots, m, \forall i=1,2, \ldots, N$,

where $V_{\mathbf{x}_{c j}}=v_{c j} V_{\mathbf{x}_{j}}$ is the corrected volume of the neighboring points $j$. As illustrated in Fig. 3.3 the approximation of the integration over the volume of the horizon size constructed for a material point $\mathbf{x}_{i}$, can be improved as [17],

$$
v_{c j}= \begin{cases}\frac{\delta+r-\xi_{i j}}{2 r} & \text { if } \delta-r<\xi_{i j}<\delta \\ 1 & \text { else }\end{cases}
$$

where $\xi_{i j}=\left|\mathbf{x}_{j}-\mathbf{x}_{i}\right|$ and $r=\Delta / 2$ is the distance from the surface of the horizon in which $\Delta$ represents the spacing between material points.

To calculate the force-vector state $\underline{\mathbf{T}}$, Silling et al. [20] proposed an approach to incorporate a classical constitutive models into the PD framework which is summarized in the flowcharts for the NOSB-PD and the force-vector state in Figs. 3 and 4, respectively. The flowchart for the JC model is shown in Fig. 5 . 


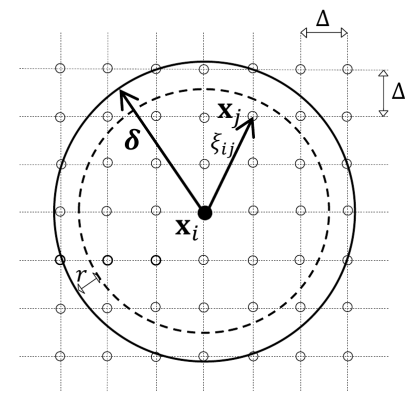

Figure 2. Volume correction for a material point inside the horizon.

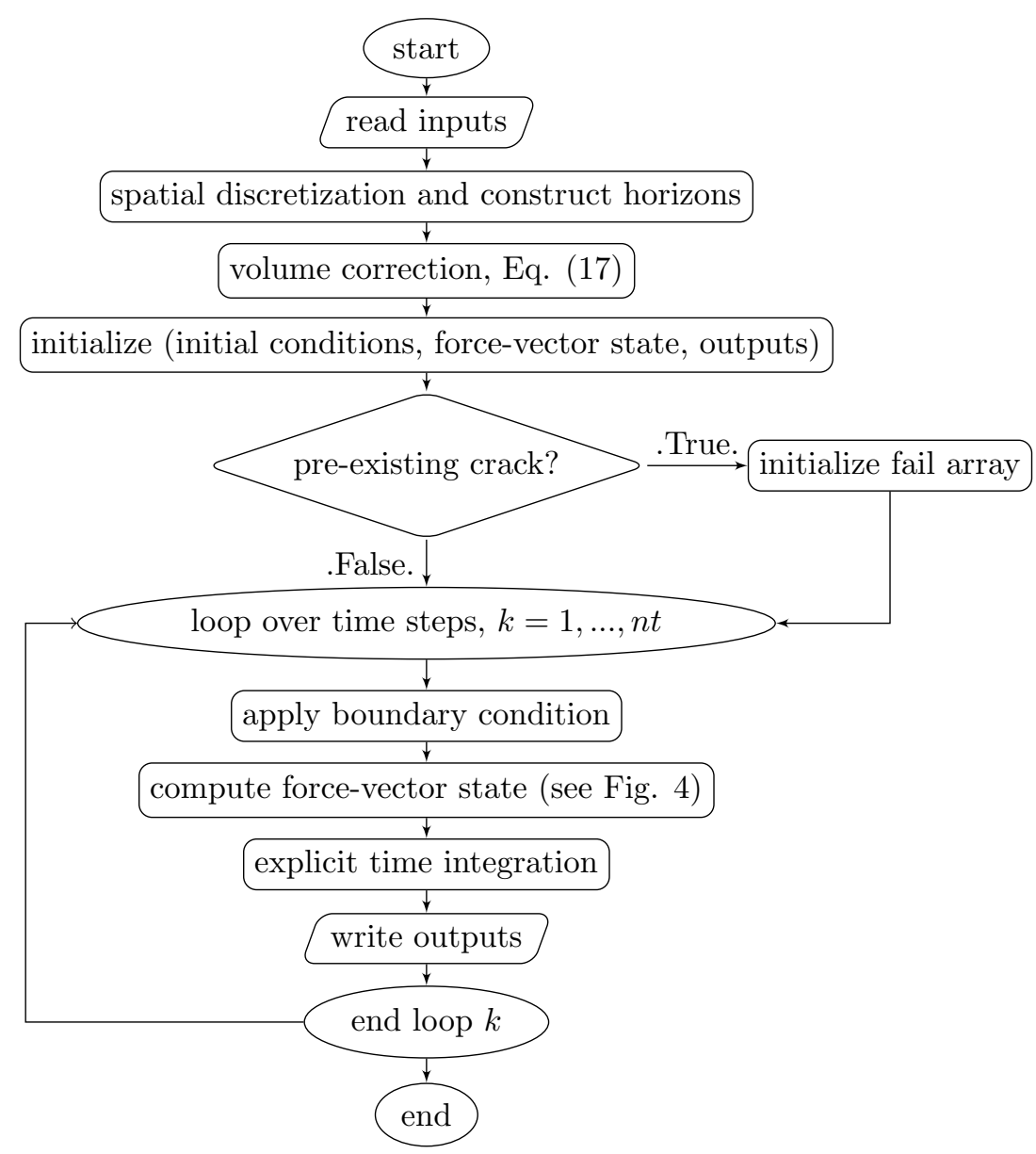

Figure 3. Flowchart of the NOSB-PD theory.

A nonlocal shape tensor is defined by a tensor product of the reference position vector state as [20],

$$
\begin{aligned}
\mathbf{K} & =\underline{\mathbf{X}}\langle\boldsymbol{\xi}\rangle * \underline{\mathbf{X}}\langle\boldsymbol{\xi}\rangle=\int_{\mathcal{H}_{\mathbf{x}_{i}}} \omega\langle\boldsymbol{\xi}\rangle(\boldsymbol{\xi} \otimes \boldsymbol{\xi}) d V_{\mathbf{x}_{j}} \approx \sum_{j=1}^{m} \omega\langle\boldsymbol{\xi}\rangle(\boldsymbol{\xi} \otimes \boldsymbol{\xi}) V_{\mathbf{x}_{c j}} \\
= & {\left[\begin{array}{ccc}
\sum_{j=1}^{m} \omega\langle\boldsymbol{\xi}\rangle \xi_{x} \xi_{x} V_{\mathbf{x}_{c j}} & \sum_{j=1}^{m} \omega\langle\boldsymbol{\xi}\rangle \xi_{x} \xi_{y} V_{\mathbf{x}_{c j}} & \sum_{j=1}^{m} \omega\langle\boldsymbol{\xi}\rangle \xi_{x} \xi_{z} V_{\mathbf{x}_{c j}} \\
& \sum_{j=1}^{m} \omega\langle\boldsymbol{\xi}\rangle \xi_{y} \xi_{y} V_{\mathbf{x}_{c j}} & \sum_{j=1}^{m} \omega\langle\boldsymbol{\xi}\rangle \xi_{y} \xi_{z} V_{\mathbf{x}_{c j}} \\
\text { symm. } & 7 & \sum_{j=1}^{m} \omega\langle\boldsymbol{\xi}\rangle \xi_{z} \xi_{z} V_{\mathbf{x}_{c j}}
\end{array}\right], }
\end{aligned}
$$




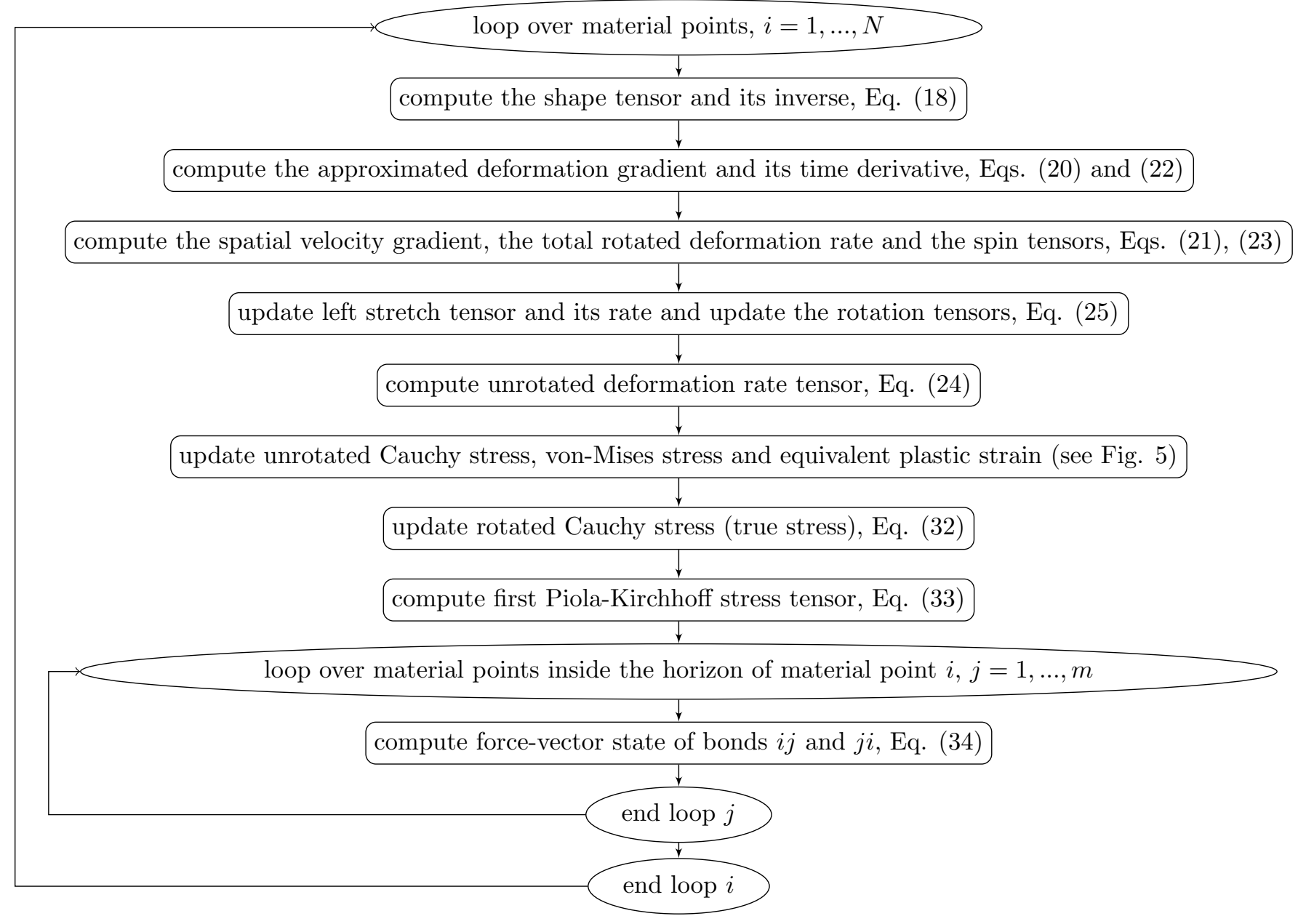

Figure 4. Flowchart for computation of the force-vector state.

where $\mathbf{K}$ is a second order, symmetric and positive definite tensor and $\omega\langle\boldsymbol{\xi}\rangle$ is the influence function.

The motion from the initial configuration $\Omega_{0}$ to the deformed configuration $\Omega$ is defined by the material deformation gradient $\mathbf{F}$ which can be expressed for the material point $i$ as

$$
\mathbf{F}=\frac{\partial \mathbf{y}\left(\mathbf{x}_{i}, t\right)}{\partial \mathbf{x}_{i}}
$$

It was shown in [20] that $\mathbf{F}$ can be approximated by

$$
\mathbf{F}=[\underline{\mathbf{Y}}\langle\boldsymbol{\xi}\rangle * \underline{\mathbf{X}}\langle\boldsymbol{\xi}\rangle] \mathbf{K}^{-1}=\left[\int_{\mathcal{H}_{\mathbf{x}_{i}}} \omega\langle\boldsymbol{\xi}\rangle(\underline{\mathbf{Y}}\langle\boldsymbol{\xi}\rangle \otimes \boldsymbol{\xi}) d V_{\mathbf{x}_{j}}\right] \mathbf{K}^{-1} \approx\left[\sum_{j=1}^{m} \omega\langle\boldsymbol{\xi}\rangle(\underline{\mathbf{Y}}\langle\boldsymbol{\xi}\rangle \otimes \boldsymbol{\xi}) V_{\mathbf{x}_{c j}}\right] \mathbf{K}^{-1} .
$$




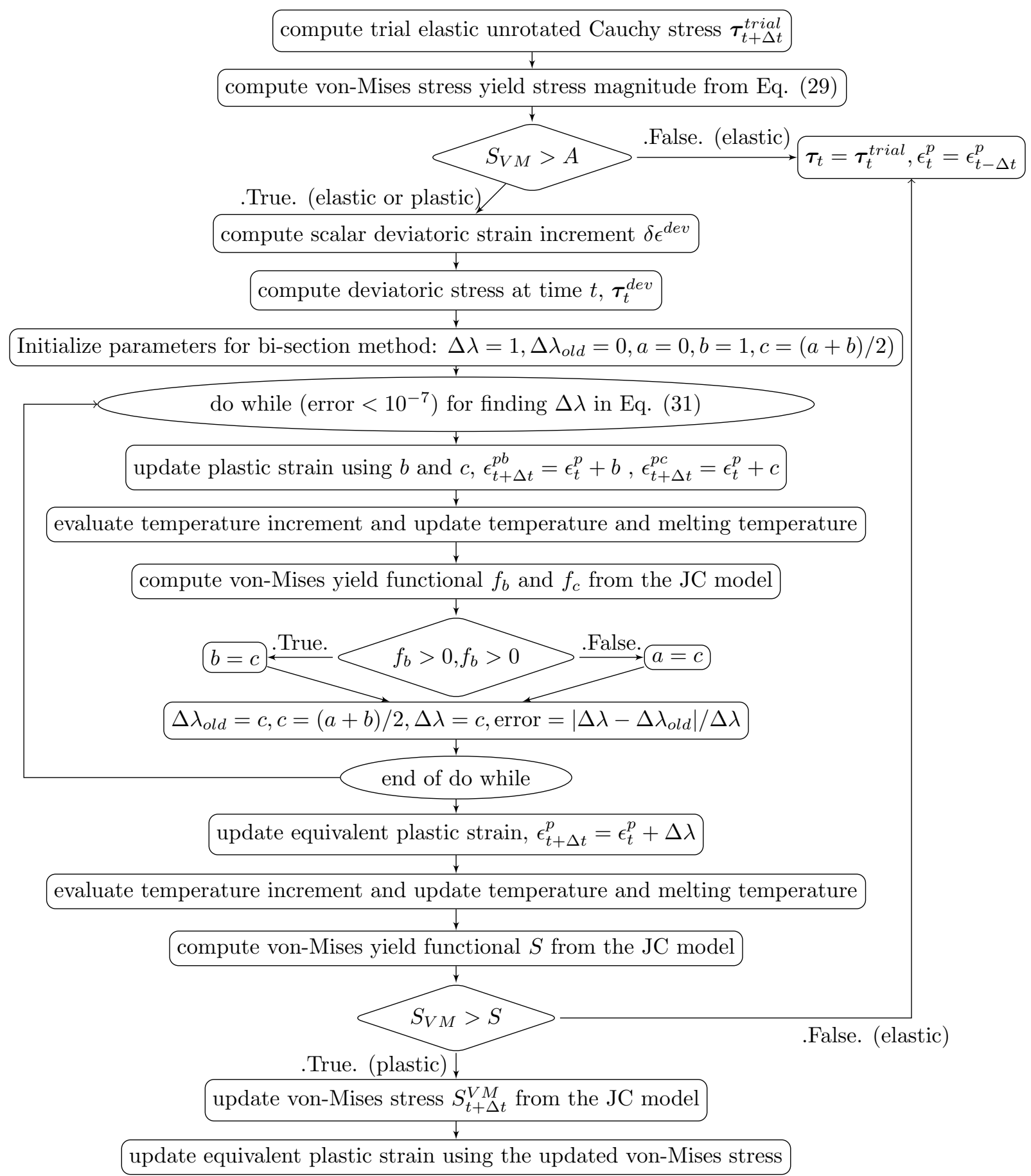

Figure 5. Flowchart of the JC model. 
The spatial velocity gradient is obtained from the well known relation

$$
\mathbf{L}=\dot{\mathbf{F}} \mathbf{F}^{-1}
$$

where $\dot{\mathbf{F}}$ is the material time derivative of $\mathbf{F}$ defined as

$$
\dot{\mathbf{F}}=\left[\int_{\mathcal{H}_{\mathbf{x}_{i}}} \omega\langle\boldsymbol{\xi}\rangle(\underline{\dot{\mathbf{Y}}}\langle\boldsymbol{\xi}\rangle \otimes \boldsymbol{\xi}) d V_{\mathbf{x}_{j}}\right] \mathbf{K}^{-1} \approx\left[\sum_{j=1}^{m} \omega\langle\boldsymbol{\xi}\rangle(\underline{\dot{\mathbf{Y}}}\langle\boldsymbol{\xi}\rangle \otimes \boldsymbol{\xi}) V_{\mathbf{x}_{c j}}\right] \mathbf{K}^{-1} .
$$

The deformation rate of tensor $\mathbf{D}$ and spin tensor $\mathbf{W}$ follows from $\mathbf{L}$ :

$$
\mathbf{D}=\operatorname{sym}(\mathbf{L})=\frac{1}{2}\left[\mathbf{L}+\mathbf{L}^{\mathrm{T}}\right], \quad \mathbf{W}=\operatorname{skew}(\mathbf{L})=\frac{1}{2}\left[\mathbf{L}-\mathbf{L}^{\mathrm{T}}\right]
$$

The unrotated deformation rate of tensor $\mathbf{d}$ can be computed as $[23,49]$

$$
\mathbf{d}=\mathbf{d}^{\mathrm{e}}+\mathbf{d}^{\mathrm{p}}+\mathbf{d}^{\mathrm{T}}=\mathbf{R}_{t}^{\mathrm{T}} \mathbf{D} \mathbf{R}_{t}
$$

where $\mathbf{d}^{\mathrm{e}}, \mathbf{d}^{\mathrm{p}}$ and $\mathbf{d}^{\mathrm{T}}$ indicate its elastic, plastic and thermal parts, respectively; $\mathbf{R}_{t}$ is an orthogonal tensor describing the rigid-body rotation at current time $t$. It is calculated in the incremental formulation as follow [50]:

$$
\mathbf{R}_{t}=\left[\mathbf{I}+\frac{\sin (\Delta t \Omega)}{\Omega} \boldsymbol{\Omega}-\frac{1-\cos (\Delta t \Omega)}{\Omega^{2}} \boldsymbol{\Omega}^{2}\right] \mathbf{R}_{t-\Delta t}
$$

where $\Omega^{2}=\omega_{k} \omega_{k}$ and $\Omega_{i j}=e_{i k j} \omega_{k}$ in which $e_{i k j}$ is the permutation tensor and $\boldsymbol{\omega}=\mathbf{w}+\left[\operatorname{trace}\left(\mathbf{V}_{t}\right) \mathbf{I}-\mathbf{V}_{t}\right]^{-1} \mathbf{z}$. The vorticity $\mathbf{w}=-\frac{1}{2} e_{i j k} W_{j k}, z_{i}=e_{i k j} D_{j m} V_{m k}$ and $\mathbf{V}_{t}=\mathbf{V}_{t-\Delta t}+\Delta t \dot{\mathbf{V}}_{\Delta t}$ is the updated left stretch tensor where $\dot{\mathbf{V}}_{\Delta t}=\mathbf{L V}_{t}-\mathbf{V}_{t} \boldsymbol{\Omega}$ is the left stretch rate tensor. At each time step, it is required to store the tensors $\mathbf{V}$ and $\mathbf{R}$.

The unrotated deformation rate of tensor $\mathbf{d}$ is used in the JC constitutive model to calculate the unrotated Cauchy stress, $\boldsymbol{\tau}$ (for more details see [23] and Fig. 5). First, we assume only an elastic deformation increment across a time step $\left(\mathbf{d}^{\mathrm{e}}=\mathbf{d}, \mathbf{d}^{\mathrm{p}}=\mathbf{0}, \mathbf{d}^{\mathrm{T}}=\mathbf{0}\right)$. Hence, the total elastic strain increment and deviatoric strain increment tensors from the von-Mises plasticity theory are calculated as

$$
\Delta \mathbf{e}=\mathbf{d} \Delta t, \quad \Delta \mathbf{e}^{\mathrm{dev}}=\Delta \mathbf{e}-\frac{1}{3} \Delta \mathbf{e} \mathbf{I}
$$

The trial unrotated Cauchy stress at time $t$ is defined as

$$
\boldsymbol{\tau}_{t}^{\mathrm{trial}}=\boldsymbol{\tau}_{t-\Delta t}+\kappa \operatorname{trace}(\Delta \mathbf{e}) \mathbf{I}+2 \mu \Delta \mathbf{e}^{\mathrm{dev}},
$$


where $\kappa$ and $\mu$ are the Lamé's constants.

The trial deviatoric stress tensor from the von-Mises plasticity theory is

$$
\mathbf{S}^{\mathrm{dev}}=\boldsymbol{\tau}_{t}^{\text {trial }}-\frac{1}{3} \operatorname{trace}\left(\boldsymbol{\tau}_{t}^{\text {trial }}\right) \mathbf{I}
$$

The magnitude of the von-Mises yield stress is defined as

$$
S_{V M}=\sqrt{\frac{3}{2}}\left|\mathbf{S}^{\mathrm{dev}}\right|
$$

where $\left|\mathbf{S}^{\mathrm{dev}}\right|=\sqrt{S_{i j}^{\mathrm{dev}} S_{i j}^{\mathrm{dev}}}$ is the magnitude of the deviatoric stress tensor.

If $S_{V M}<A$ the material response is elastic and the unrotated Cauchy stress tensor is equal to the trial unrotated Cauchy stress tensor. Otherwise we have to compute the value of the von-Mises yield stress from the JC constitutive model. For the JC model it is required to find in the equivalent plastic strain increment by solving the following equation [23]

$$
\Delta e-\Delta \lambda-\frac{1}{2 \mu}\left[\sqrt{\frac{2}{3}} f_{y}-S_{t-\Delta t}\right]=0,
$$

where $\Delta \lambda$ is used to extent the yield surface and find the updated equivalent plastic strain and $f_{y}$ is the yield function from the constitutive model.

By substituting the yield function of the JC constitutive model

$$
\Delta e-\Delta \lambda-\frac{1}{2 \mu}\left[\sqrt{\frac{2}{3}}\left[(1-D)\left[A+B \epsilon_{r p}^{n}\right]\left[1+\dot{\epsilon}_{r p}^{*}\right]^{C}\left[1-T^{* m}\right]\right]-S_{t-\Delta t}\right]=0,
$$

To find $\Delta \lambda$, this equation should be solved with a numerical root solver (e.g. Newton-Raphson method, bi-section method). In this study the bi-section iterative solver is employed and its procedure is shown in Fig. 5.

Then, the rotated Cauchy stress tensor $\boldsymbol{\sigma}$ is obtained as follow

$$
\boldsymbol{\sigma}=\mathbf{R}_{t} \tau \mathbf{R}_{t}^{\mathrm{T}}
$$

From classical continuum theory the first Piola-Kirchhoff stress tensor, $\mathbf{P}$ is a function of the deformation gradient and the rotated Cauchy stress tensor as

$$
\mathbf{P}=(J \boldsymbol{\sigma}) \mathbf{F}^{-\mathrm{T}}
$$


Finally, the force-vector state of a bond with the bond vector $\boldsymbol{\xi}$ can be calculated as

$$
\mathbf{T}\langle\boldsymbol{\xi}\rangle=\omega\langle\boldsymbol{\xi}\rangle \mathbf{P K}^{-1} \boldsymbol{\xi}
$$

\section{Numerical results}

We validate our NOSB-PD formulation of thermoplasticity by two benchmark examples: 1) The Taylorbar impact test [51] made of Oxygen-Free High-thermal Conductivity (OFHC) Copper specimen and 2) the Kalthoff-Winkler test $[52,53,54]$ made of steel. 3D numerical simulations are performed based on the initial dimensions, initial velocity and physical properties presented in Table 1. The numerical simulations are compared with available experimental results. For all simulations, the horizon size is $\delta=3.015 \Delta$ where $\Delta$ is mean distance of the material points along $x, y$ and $z$ directions.

Table 1. Physical properties of OFHC-Copper and 4340 Steel specimens[35, 36].

\begin{tabular}{|c|c|c|c|c|}
\hline & & Unit & OFHC-Copper & 4340 Steel \\
\hline \multicolumn{5}{|l|}{ Initial Dimensions and velocity: } \\
\hline Initial length & $L_{0}$ & $\mathrm{~mm}$ & 25.4 & 100 \\
\hline Initial cylinder diameter (Taylor-bar impact test) & $D_{0}$ & $\mathrm{~mm}$ & 7.62 & - \\
\hline Initial width (KW test) & $W_{0}$ & $\mathrm{~mm}$ & - & 200 \\
\hline Initial thickness (KW test) & $t_{0}$ & $\mathrm{~mm}$ & - & 6.35 \\
\hline Impact velocity & $V_{0}$ & $\mathrm{~m} / \mathrm{s}$ & 190 & 32 \\
\hline \multicolumn{5}{|l|}{ Material constants: } \\
\hline Elastic modulus & $E$ & $\mathrm{GPa}$ & 124 & 200 \\
\hline Poisson's ratio & $\nu$ & - & 0.34 & 0.29 \\
\hline Mass density & $\rho$ & $\mathrm{kg} / \mathrm{m}^{3}$ & 8960 & 7830 \\
\hline Thermal conductivity & $\kappa$ & $\mathrm{W} / \mathrm{mK}$ & 386 & 38 \\
\hline Specific heat capacity & $C_{p}$ & $\mathrm{~J} / \mathrm{kgK}$ & 383 & 477 \\
\hline Thermal expansion coefficient & $\alpha$ & $1 / \mathrm{K}$ & 0.00005 & 0.000032 \\
\hline Reference (room) temperature & $T_{0}$ & $\mathrm{~K}$ & 298 & 298 \\
\hline Melting temperature & $T_{m}$ & $\mathrm{~K}$ & 1356 & 1793 \\
\hline Taylor-Quinney coefficient & $\beta$ & - & 0.9 & 0.9 \\
\hline Reference equivalent plastic strain rate & $\dot{\epsilon}_{p_{0}}$ & $1 / \mathrm{s}$ & 1.0 & 1.0 \\
\hline \multicolumn{5}{|l|}{ Strength constants: } \\
\hline Yield stress & $A$ & $\mathrm{MPa}$ & 90 & 792 \\
\hline Strain hardening constant & $B$ & $\mathrm{MPa}$ & 292 & 510 \\
\hline Strain hardening coefficient & $n$ & - & 0.31 & 0.26 \\
\hline Strain rate constant & $C$ & - & 0.025 & 0.014 \\
\hline Thermal softening exponent parameter & $m$ & - & 1.09 & 1.03 \\
\hline \multicolumn{5}{|l|}{ Fracture constants: } \\
\hline & $D_{1}$ & - & 0.54 & 0.05 \\
\hline & $D_{2}$ & - & 4.89 & 3.44 \\
\hline & $D_{3}$ & - & -3.03 & -2.12 \\
\hline & $D_{4}$ & - & 0.014 & 0.002 \\
\hline & $D_{5}$ & - & 1.12 & 0.61 \\
\hline
\end{tabular}



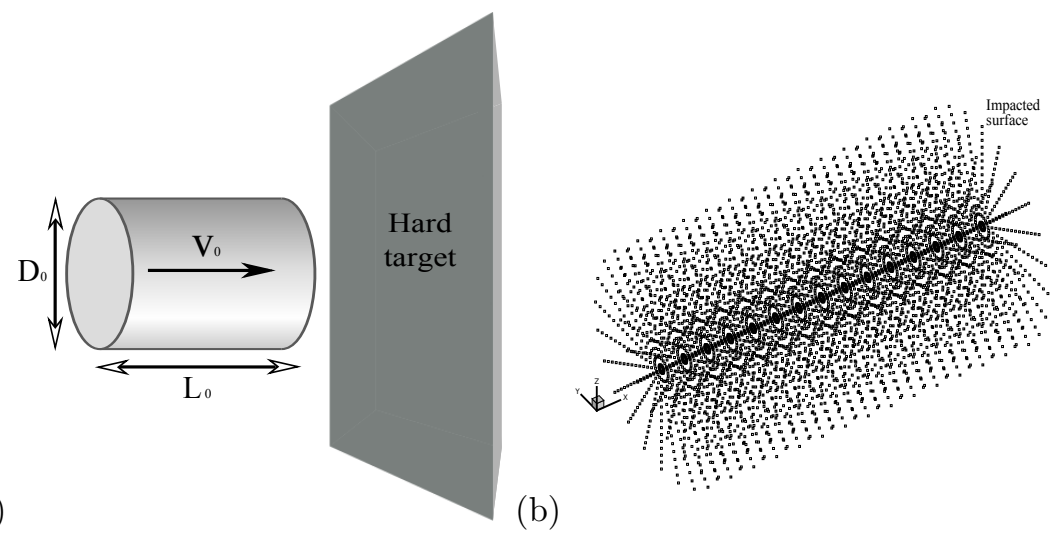

Figure 6. (a) The Taylor-bar impact test, (b) a material points distribution with 5,400 nodes.

\subsection{The Taylor-bar impact test on an OFHC-Copper specimen}

In the Taylor-bar impact test, a cylindrical projectile is shot on a hard target (see Fig. 6-(a)). As described by Whiffin [55], this test is designed for relatively small deformations obtained from low velocity impacts. Though the Taylor-bar impact test continues to be used to determine yield strengths of materials at high strain rates, it is limited to peak strains around 0.6 at the center of the specimen [56]. For higher strains and strain rates, the Taylor-bar impact test is mostly used for validating plasticity models.

Fig. 6-(b) shows initial material points distribution with 5,400 nodes. The PD simulations are done for $32.5 \mu \mathrm{sec}$. The time step size is chosen as $0.1 \mu \mathrm{sec}$ which leads to 325 time steps. In Fig. 7 the contours of the displacement in $y$ direction, temperature, von-Mises stress and equivalent plastic strain of the bar are plotted at different time steps. There is a good agreement between the PD and the experimental dynamic structured light (DSL) image results [57] and the mushroom shape is captured successfully in comparison with the experimental test. The variation of the homologous temperature due to the plastic work versus time at different material points is plotted in Fig. 4.1-(a). These material points are selected from different layers in length direction with the same coordinates along $y$ and $z$ directions to show the variation of the temperature with distance from the impacted surface. The change in the homologous temperature of a material point only depends on the change in the current temperature which confirms Eq. (2).

Based on the fracture constants of the OFHC-Copper specimen presented in Table 1 the accumulated damage is calculated and used in the PD formulation of the JC model. The accumulated damage changes with variation of the equivalent plastic strain and homologous temperature. Fig. 4.1-(b) shows the variation of thermal softening fraction of the JC damage model versus homologous temperature. The slope of this variation depends on the thermal softening exponent parameter $m$ in which for the OFHC-Copper specimen is 1.09 . 
(Exp.)
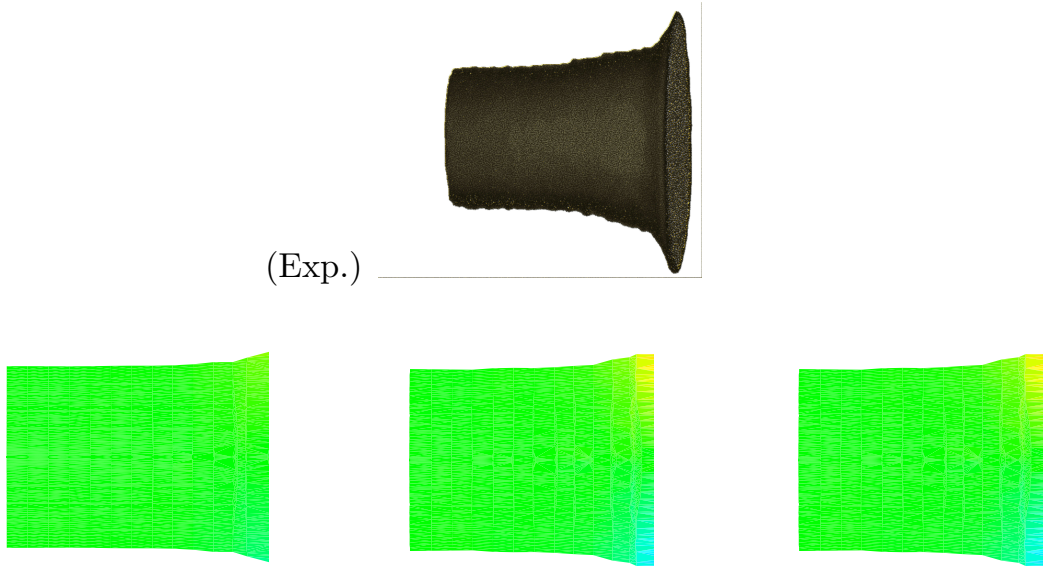

I:(a)

(b)

(c)

$0.0016 \quad 0.0025 \quad 0.0036$

(b)

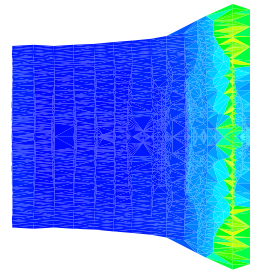

II:(a)

(c)

$298 \quad 421 \quad 543 \quad 666 \quad 788 \quad 911 \quad 1034 \quad 1156 \quad 1279 \quad 1401 \quad 1524164617691890$
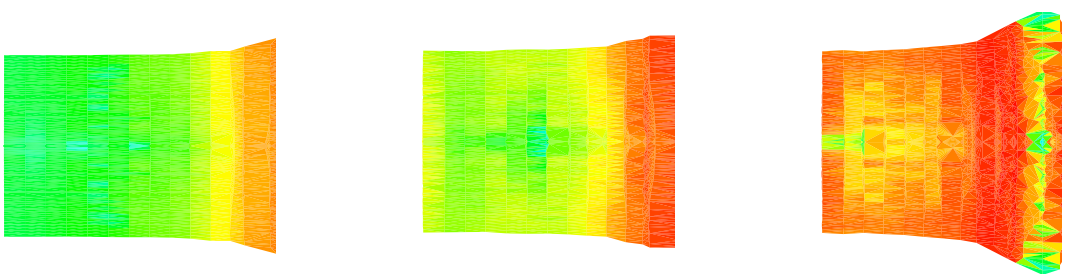

III:(a)

(b)

(c)

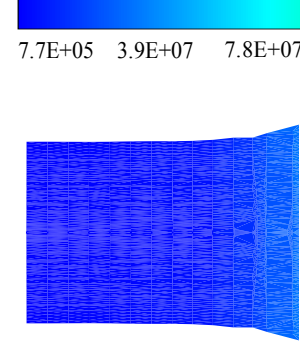

$\mathrm{E}+08$

$1.5 \mathrm{E}+08$

$1.9 \mathrm{E}+08$

$3 \mathrm{E}+08 \quad 2.7 \mathrm{E}+08 \quad 3.1 \mathrm{E}+08$

IV:(a)

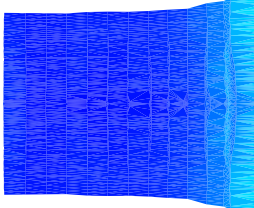

(b)

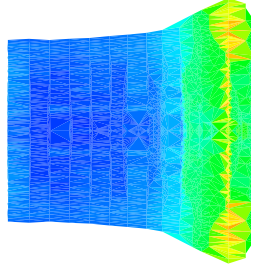

(c)

$\begin{array}{lllllllllll}0.0 & 0.245 & 0.507 & 0.768 & 1.030 & 1.292 & 1.553 & 1.815 & 2.077 & 2.338 & 2.6\end{array}$

Figure 7. (Exp.) Post test deformation from dynamic structural light (DSL) image [57], Contours of (I) displacement in $y$ direction, (II) temperature, (III) von-Mises stress and (IV) equivalent plastic strain, at time steps (a) 80 , (b) 160 and (c) 325. 
(a)

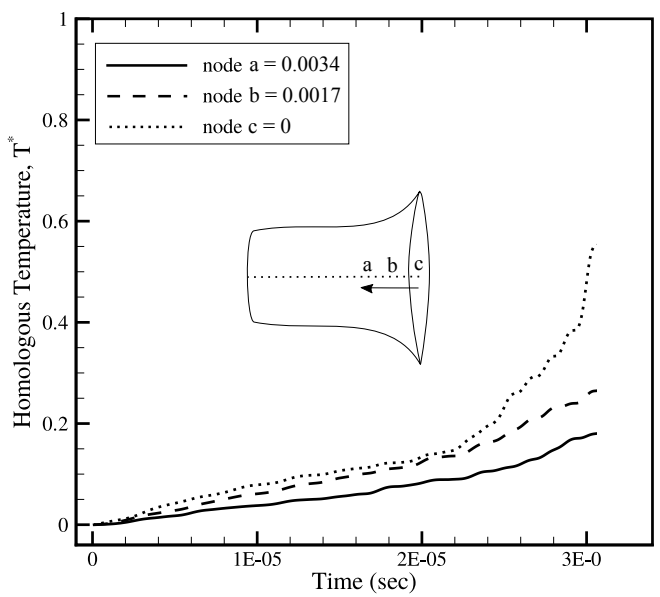

(b)

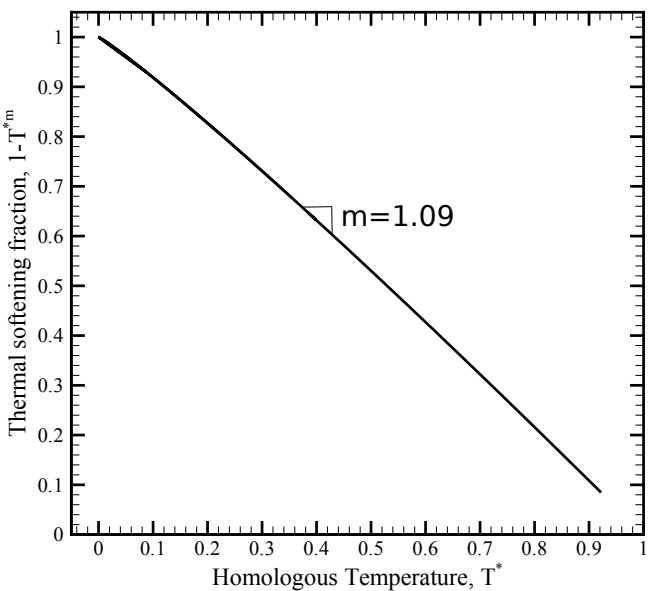

Figure 8. Variation of (a) homologous temperature versus time, (b) thermal softening fraction of the JC damage model versus homologous temperature.

\subsection{The Kalthoff-Winkler test on a 4340 Steel specimen}

Kalthoff and Winkler performed a series of experiments $[52,53,54]$ where a double notched steel plate is subjected to impact loading as shown in Fig. 9-(a). They discovered that the failure mode depends on the impact velocity. At low impact velocity the specimen failed due to a mode I dominated crack propagation in an angle of approximately $70^{\circ}$ counterclockwise with respect to the notch-axis. However, at higher impact speeds, a shear-band initiated from a point on the lower surface of the notch-tip and propagated at an angle of $10^{0}$ clockwise from the axis of the notch, i.e., towards the impacted part of the plate. As shown in Fig. 9-(b), the material points distribution with 60,291 nodes is used for discretization of the problem domain.

plate $=6.35 \mathrm{~mm}$

Radius of each notch $=0.15 \mathrm{~mm}$

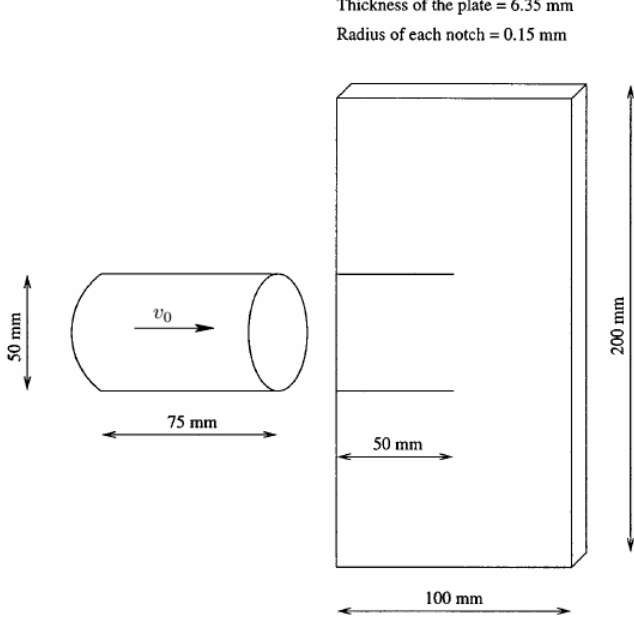

(a)

Figure 9. (a) The Kalthoff-Winkler (KW) test, (b) a material points distribution with 60,291 nodes.

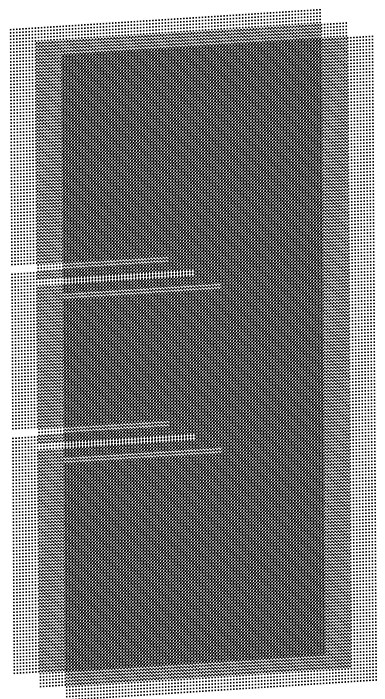

(b) 


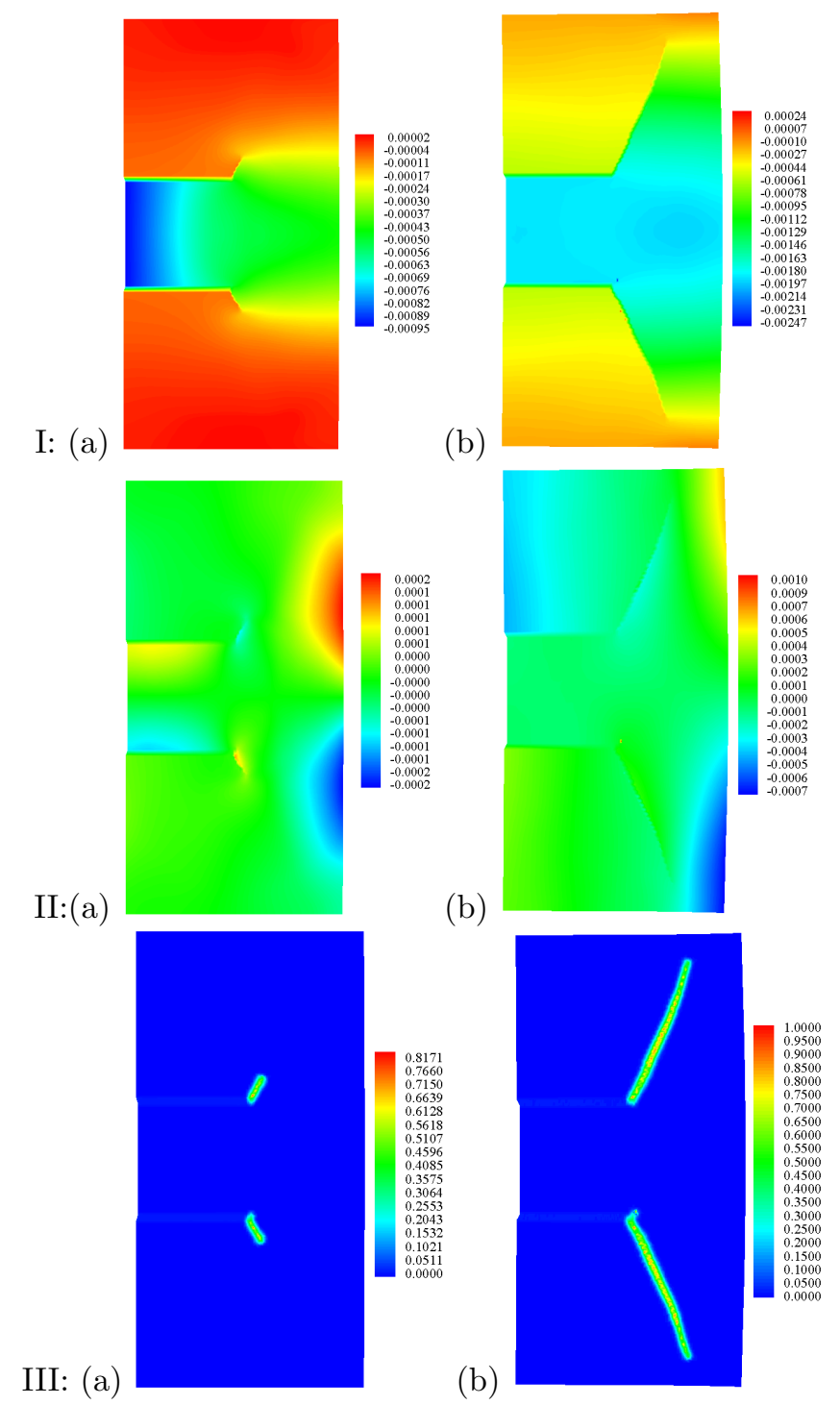

Figure 10. Contours of (I) displacement in $x$ direction, (II) displacement in $y$ direction and (III) damage on the deformed shape at time steps (a) 400, (b) 800.

Fig. 10 shows the contours of displacement in $x$ and $y$ directions and the damage on the deformed shape of the surface $x y$ at different time steps. The brittle crack propagates at an angle of $70^{0}$ from the axis of notches matching well with the experimental observations.

\section{Conclusions}

A non-ordinary state-based peridynamics formulation was developed for fracture analysis of thermomechanical problems. The Johnson-Cook constitutive and damage models was used. The numerical results agreed well with the available experimental data. We will extend this model to consider effect of friction and 
erosion in high temperature impact tests. Also, different ductile damage models such as Gurson-TvergaardNeedleman (GTN) and Rousselier model will be implemented. In addition, the fully coupled formulation of thermomechanical problems with the JC model will be investigated in future works to find coupling effect of strain rate and temperature on the results.

\section{Acknowledgement}

This work was supported by European Union through ERC grant for COMBAT project is greatly acknowledged by the first author.

\section{References}

[1] N. Moes, J. Dolbow, T. Belytschko, A finite element method for crack growth without remeshing, International journal for numerical methods in engineering 46 (1) (1999) 131-150.

[2] A. Daneshyar, S. Mohammadi, Strong tangential discontinuity modeling of shear bands using the extended finite element method, Computational Mechanics 52 (5) (2013) 1023-1038.

[3] S. Li, W.-K. Liu, D. Qian, P. R. Guduru, A. J. Rosakis, Dynamic shear band propagation and micro-structure of adiabatic shear band, Computer Methods in Applied Mechanics and Engineering 191 (1) (2001) 73-92.

[4] S. Li, W. K. Liu, A. J. Rosakis, T. Belytschko, W. Hao, Mesh-free galerkin simulations of dynamic shear band propagation and failure mode transition, International Journal of solids and structures 39 (5) (2002) 1213-1240.

[5] T. Rabczuk, P. Areias, T. Belytschko, A simplified mesh-free method for shear bands with cohesive surfaces, International Journal for Numerical Methods in Engineering 69 (5) (2007) 993-1021.

[6] T. Rabczuk, T. Belytschko, A three-dimensional large deformation meshfree method for arbitrary evolving cracks, Computer Methods in Applied Mechanics and Engineering 196 (29) (2007) 2777-2799.

[7] T. Rabczuk, P. Areias, T. Belytschko, A meshfree thin shell method for non-linear dynamic fracture, International Journal for Numerical Methods in Engineering 72 (5) (2007) 524-548.

[8] X. Zhuang, C. Augarde, K. Mathisen, Fracture modeling using meshless methods and level sets in 3d: framework and modeling, International Journal for Numerical Methods in Engineering 92 (11) (2012) 969-998.

[9] X. Zhuang, Y. Cai, C. Augarde, A meshless sub-region radial point interpolation method for accurate calculation of crack tip fields, Theoretical and Applied Fracture Mechanics 69 (2014) 118-125.

[10] J.-H. Song, P. Areias, T. Belytschko, A method for dynamic crack and shear band propagation with phantom nodes, International Journal for Numerical Methods in Engineering 67 (6) (2006) 868-893.

[11] T. Chau-Dinh, G. Zi, P.-S. Lee, T. Rabczuk, J.-H. Song, Phantom-node method for shell models with arbitrary cracks, Computers \& Structures 92 (2012) 242-256.

[12] N. Vu-Bac, H. Nguyen-Xuan, L. Chen, C.-K. Lee, G. Zi, X. Zhuang, G. R. Liu, T. Rabczuk, A phantom-node method with edge-based strain smoothing for linear elastic fracture mechanics, Journal of Applied Mathematics 2013.

[13] S. S. Ghorashi, N. Valizadeh, S. Mohammadi, T. Rabczuk, T-spline based xiga for fracture analysis of orthotropic media, Computers \& Structures 147 (2015) 138-146.

[14] N. Nguyen-Thanh, N. Valizadeh, M. Nguyen, H. Nguyen-Xuan, X. Zhuang, P. Areias, G. Zi, Y. Bazilevs, L. De Lorenzis, T. Rabczuk, An extended isogeometric thin shell analysis based on kirchhoff-love theory, Computer Methods in Applied Mechanics and Engineering 284 (2015) 265-291. 
[15] P. Areias, T. Rabczuk, P. Camanho, Initially rigid cohesive laws and fracture based on edge rotations, Computational Mechanics 52 (4) (2013) 931-947.

[16] P. Areias, T. Rabczuk, P. Camanho, Finite strain fracture of 2d problems with injected anisotropic softening elements, Theoretical and Applied Fracture Mechanics 72 (2014) 50-63.

[17] E. Madenci, E. Oterkus, Peridynamic theory and its applications, Springer, 2014.

[18] A. C. Eringen, Nonlocal continuum field theories, Springer, 2002.

[19] S. A. Silling, Reformulation of elasticity theory for discontinuities and long-range forces, Journal of the Mechanics and Physics of Solids 48 (1) (2000) 175-209.

[20] S. A. Silling, M. Epton, O. Weckner, J. Xu, E. Askari, Peridynamic states and constitutive modeling, Journal of Elasticity 88 (2) (2007) 151-184.

[21] J. A. Mitchell, A nonlocal, ordinary, state-based plasticity model for peridynamics.

[22] T. L. Warren, S. A. Silling, A. Askari, O. Weckner, M. A. Epton, J. Xu, A non-ordinary state-based peridynamic method to model solid material deformation and fracture, International Journal of Solids and Structures 46 (5) (2009) $1186-1195$.

[23] J. Foster, S. A. Silling, W. Chen, Viscoplasticity using peridynamics, International journal for numerical methods in engineering 81 (10) (2010) 1242-1258.

[24] M. Tupek, J. Rimoli, R. Radovitzky, An approach for incorporating classical continuum damage models in state-based peridynamics, Computer Methods in Applied Mechanics and Engineering 263 (2013) 20-26.

[25] B. Kilic, E. Madenci, Prediction of crack paths in a quenched glass plate by using peridynamic theory, International journal of fracture 156 (2) (2009) 165-177.

[26] F. Bobaru, M. Duangpanya, The peridynamic formulation for transient heat conduction, International Journal of Heat and Mass Transfer 53 (19) (2010) 4047-4059.

[27] F. Bobaru, M. Duangpanya, A peridynamic formulation for transient heat conduction in bodies with evolving discontinuities, Journal of Computational Physics 231 (7) (2012) 2764-2785.

[28] B. Kilic, E. Madenci, Peridynamic theory for thermomechanical analysis, Advanced Packaging, IEEE Transactions on 33 (1) (2010) 97-105.

[29] W. Gerstle, S. A. Silling, D. Read, V. Tewary, R. Lehoucq, Peridynamic simulation of electromigration, Comput Mater Continua 8 (2) (2008) 75-92.

[30] S. Oterkus, E. Madenci, A. Agwai, Peridynamic thermal diffusion, Journal of Computational Physics 265 (2014) $71-96$.

[31] S. Oterkus, J. Fox, E. Madenci, Simulation of electro-migration through peridynamics, in: Electronic Components and Technology Conference (ECTC), 2013 IEEE 63rd, IEEE, 2013, pp. 1488-1493.

[32] A. G. Agwai, A peridynamic approach for coupled fields, Ph.D. thesis (2011).

[33] S. Oterkus, E. Madenci, A. Agwai, Fully coupled peridynamic thermomechanics, Journal of the Mechanics and Physics of Solids 64 (2014) 1-23.

[34] D. Simkins, S. Li, Meshfree simulations of thermo-mechanical ductile fracture, Computational Mechanics 38 (3) (2006) $235-249$.

[35] G. R. Johnson, W. H. Cook, A constitutive model and data for metals subjected to large strains, high strain rates and high temperatures, in: Proceedings of the 7th International Symposium on Ballistics, Vol. 21, The Netherlands, 1983, pp. $541-547$.

[36] G. R. Johnson, W. H. Cook, Fracture characteristics of three metals subjected to various strains, strain rates, temperatures and pressures, Engineering fracture mechanics 21 (1) (1985) 31-48.

[37] G. Camacho, M. Ortiz, Adaptive lagrangian modelling of ballistic penetration of metallic targets, Computer methods in applied mechanics and engineering 142 (3) (1997) 269-301.

[38] X. Wang, J. Shi, Validation of johnson-cook plasticity and damage model using impact experiment, International Journal 
of Impact Engineering 60 (2013) 67-75.

[39] T. Børvik, O. Hopperstad, T. Berstad, M. Langseth, A computational model of viscoplasticity and ductile damage for impact and penetration, European Journal of Mechanics-A/Solids 20 (5) (2001) 685-712.

[40] G. I. Taylor, H. Quinney, The latent energy remaining in a metal after cold working, Proceedings of the Royal Society of London. Series A 143 (849) (1934) 307-326.

[41] N. Jones, Dynamic inelastic failure of structures., A 63 (616) (1997) 2485-2495.

[42] M. Zhou, G. Ravichandran, A. Rosakis, Dynamically propagating shear bands in impact-loaded prenotched platesii. numerical simulations, Journal of the Mechanics and Physics of Solids 44 (6) (1996) 1007-1032.

[43] C. McAuliffe, H. Waisman, Mesh insensitive formulation for initiation and growth of shear bands using mixed finite elements, Computational Mechanics 51 (5) (2013) 807-823.

[44] C. McAuliffe, H. Waisman, A pian-sumihara type element for modeling shear bands at finite deformation, Computational Mechanics 53 (5) (2014) 925-940.

[45] L. Berger-Vergiat, C. McAuliffe, H. Waisman, Isogeometric analysis of shear bands, Computational Mechanics 54 (2) (2014) 503-521

[46] F. Armero, J. Simo, A new unconditionally stable fractional step method for non-linear coupled thermomechanical problems, International Journal for numerical methods in Engineering 35 (4) (1992) 737-766.

[47] F. Armero, J. Simo, A priori stability estimates and unconditionally stable product formula algorithms for nonlinear coupled thermoplasticity, International Journal of Plasticity 9 (6) (1993) 749-782.

[48] R. Smerd, S. Winkler, C. Salisbury, M. Worswick, D. Lloyd, M. Finn, High strain rate tensile testing of automotive aluminum alloy sheet, International Journal of Impact Engineering 32 (1) (2005) 541-560.

[49] A. Needleman, V. Tvergaard, Analysis of a brittle-ductile transition under dynamic shear loading, International Journal of Solids and Structures 32 (17) (1995) 2571-2590.

[50] D. Flanagan, L. Taylor, An accurate numerical algorithm for stress integration with finite rotations, Computer methods in applied mechanics and engineering 62 (3) (1987) 305-320.

[51] G. Taylor, The use of flat-ended projectiles for determining dynamic yield stress. i. theoretical considerations, Proceedings of the Royal Society of London. Series A. Mathematical and Physical Sciences 194 (1038) (1948) 289-299.

[52] J. F. Kalthoff, Shadow optical analysis of dynamic shear fracture, Optical Engineering 27 (10) (1988) 271035-271035.

[53] J. Kalthoff, S. Winkler, Failure mode transition at high rates of shear loading, DGM Informationsgesellschaft mbH, Impact Loading and Dynamic Behavior of Materials 1 (1988) 185-195.

[54] J. F. Kalthoff, Modes of dynamic shear failure in solids, International Journal of Fracture 101 (1-2) (2000) 1-31.

[55] A. Whiffin, The use of flat-ended projectiles for determining dynamic yield stress. ii. tests on various metallic materials, Proceedings of the Royal Society of London. Series A. Mathematical and Physical Sciences 194 (1038) (1948) 300-322.

[56] G. R. Johnson, T. J. Holmquist, Evaluation of cylinder-impact test data for constitutive model constants, Journal of Applied Physics 64 (8) (1988) 3901-3910.

[57] C. E. Anderson Jr, A. E. Nicholls, I. S. Chocron, R. A. Ryckman, Taylor anvil impact, in: SHOCK COMPRESSION OF CONDENSED MATTER-2005: Proceedings of the Conference of the American Physical Society Topical Group on Shock Compression of Condensed Matter, Vol. 845, AIP Publishing, 2006, pp. 1367-1370. 